\title{
Voluntariado: benefício a quem presta e a quem recebe
}

\author{
Volunteerism: benefits for both providers and recipients
}

\author{
Alexander Sapiro $₫$ $\bowtie$, Rita Mattiello ${ }^{2}$ \\ ${ }^{1}$ Professor e Coordenador dos Projetos de Voluntariado da Escola de Medicina da Pontifícia Universidade Católica do Rio Grande do Sul (PUCRS). Porto Alegre, RS. \\ 2 Professora da Escola de Medicina e do Programa de Pós-Graduação em Pediatria e Saúde da Criança da PUCRS. Porto Alegre, RS.
}

\section{RESUMO}

Objetivos: É importante que durante o curso de medicina não seja negligenciada a atenção aos aspectos humanos e sociais da profissão. O presente artigo aborda o voluntariado direcionado aos aspectos sociais, com enfoque nas ações desenvolvidas por estudantes de medicina, nos quais se almeja despertar atitudes de generosidade e beneficência.

Métodos: $\mathrm{O}$ artigo consiste em revisão da literatura, relato de experiência e reflexões sobre as atividades de voluntariado, abordando os efeitos que esse tipo de atividade pode trazer para os envolvidos.

Resultados: Voluntariado caracteriza-se pelo ato de doar tempo e conhecimento em prol da sociedade em que se vive, com ações não remuneradas, de valor para a comunidade ou o próximo. Para a Organização das Nações Unidas, voluntário é alguém que, por interesse pessoal ou espírito cívico, dedica parte do tempo a atividades que visam o bem-estar social ou utilidade pública, sem remuneração. A atividade de voluntariado representa um ato de generosidade, de beneficência para com os outros, principalmente os mais necessitados. É desejável que estudantes se tornem mais abertos para as diferenças e generosos nas relações humanas em suas futuras atividades profissionais. Além disso, pesquisas mostram que os atos de altruísmo, bondade e generosidade ativam áreas do cérebro responsáveis pela liberação de endorfina nas pessoas que os praticam, aumentando a percepção de felicidade e diminuindo o nível de estresse e ansiedade, além de trazer outros efeitos fisiológicos, como diminuição dos níveis tensionais e melhora da função imunológica. Apesar desses benefícios, cabe a reflexão sobre o quanto estamos dispostos a considerar o voluntariado como uma atividade oficialmente curricular nas escolas de medicina.

Conclusões: Atividades de voluntariado durante o curso de medicina podem proporcionar ao estudante o contato direto com comunidades e pessoas, especialmente aquelas em condições de vulnerabilidade social, promovendo um maior envolvimento humano e trazendo benefícios mútuos, a quem presta e a quem recebe. Poder ajudar e permitir que sejamos ajudados pode abrir espaços, criar redes de colaboração e solidariedade, condições ou virtudes necessárias ao bom desempenho da medicina.

DESCRITORES: trabalho voluntário; voluntariado; beneficência; estudantes de medicina; currículo.

\section{ABSTRACT}

Aims: It is paramount that attention to human and social aspects of the medical profession not be overlooked in medical schools. The present article deals with volunteerism and its social aspects, focusing on the actions developed by medical students, with the aim of arousing their generosity and beneficence.

Methods: This article consists of a literature review, report on practical experience, and reflections about volunteer activities, looking into its effects on the participants.

Results: Volunteerism is characterized by giving one's time and knowledge in the interest of the society in which one lives, on an unpaid basis, attaching importance to the community or to the others. According to the United Nations Organization, a volunteer is someone who, out of personal interest or citizenship, devotes some time to activities aimed at social welfare or public services, without any remuneration Volunteerism allows exercising generosity and beneficence towards others, especially towards the neediest. It is desirable that students become more open to differences and be generous in their future professional activities. Furthermore, research has shown that selflessness, kindheartedness, and generosity activate areas of the brain that release endorphins, thus increasing the sensation of happiness and reducing stress and anxiety, in addition to other physiological effects, such as reduction of tension and improvement of immunological function Notwithstanding these benefits, it is necessary to give some thought to how willing one is to regard volunteerism as an officially curricular activity in medical schools.

Conclusions: Volunteer activities in medical schools can offer students direct contact with communities and people, especially with socially vulnerable individuals, promoting greater human involvement and bringing benefits for both providers and recipients of volunteer work. Being able to help and allowing being helped can open up opportunities and build collaboration and solidarity networks, which are conditions or virtues necessary for the good practice of medicine.

KEY WORDS: volunteering; volunteerism; beneficence; students, medical; curriculum.

Recebido: setembro, 2016

Aceito: novembro, 2016 


\section{INTRODUÇÃO}

Com o rápido e crescente avanço de novas técnicas e equipamentos que a cada dia se agregam ao exercício da medicina, a relação entre médico e paciente corre o risco de ficar em plano secundário. $\mathrm{O}$ fácil acesso à tecnologia pode induzir o profissional a apenas avaliar os resultados de exames laboratoriais ou de imagens, com pouca ênfase na atenção, zelo e cuidados com a pessoa do paciente e seu respectivo núcleo familiar e social.

Assim, é importante que durante o curso de medicina não seja negligenciada a atenção aos aspectos humanos e sociais da profissão. Entre as ações propostas com esta finalidade, destacam-se as atividades de voluntariado. $O$ presente artigo pretende focar o voluntariado direcionado aos aspectos sociais, ou seja, que almeja despertar nos estudantes a generosidade e a beneficência, ao se relacionarem com pessoas em condições, quase sempre, bem diferentes da realidade de suas próprias famílias.

\section{VOLUNTARIADO, GENEROSIDADE E BENEFICÊNCIA}

O voluntariado é caracterizado pelo ato de uma pessoa doar seu tempo e conhecimento em prol da sociedade em que vive, com ações não remuneradas, de valor para a comunidade ou o próximo. Tem origem no latim voluntariu (fazer ou deixar de fazer, espontaneamente, sem coação nem imposição de ninguém) [1]. Portanto, a atividade de voluntariado possibilita, àquele que a pratica, um ato de generosidade, de beneficência para com os outros, principalmente os mais necessitados.

A Organização das Nações Unidas (ONU) regulamentou o programa de voluntariado em 1970 pela resolução 2659 da Assembleia Geral, com posterior criação da UNV (United Nations Volunteers), com sede em Bonn, Alemanha. Tem o dia 5 de dezembro como Dia Internacional do Voluntariado. Para a ONU, voluntário é alguém que, independentemente da idade, seja por interesse pessoal ou espírito cívico, dedica parte de seu tempo a atividades organizadas ou não, visando o bem-estar social ou algum outro campo de utilidade pública, sem remuneração. $\mathrm{O}$ voluntário da atualidade não se limita a socorrer os necessitados, mas busca a promoção de mudanças e transformação social, transcende os interesses individuais e se coloca no campo da cidadania, uma preocupação não apenas com o "eu", mas com o "outro" e o mundo [2].
A generosidade distingue-se por gratidão, amor, respeito, alegria e esperança. A pessoa generosa é aquela que consegue fazer o bem, isto é, promover a beneficência [1]. Segundo Sussman e Cloninger [3], não existe generosidade nata no ser humano e, por isso, é tão difícil esse atributo ser difundido de um modo preciso ou padronizado. Essas ideias e conceitos fazem parte da socialização do ser humano, que aprende a se comportar de acordo com sua cultura. A maneira como a pessoa age depende de sua experiência de aprendizado em sociedade [3]. Van Dyken e Wade [4,5] ponderam que o ambiente define o modo como se ajuda o próximo, e isso pode variar de sociedade para sociedade, dependendo de fatores genéticos e outros. Em seu livro "O Conselheiro", Burg e Mann [6] dizem que um profissional generoso tem mais chance de ser bem-sucedido, mas diferenciam generosidade de caridade. Destacam ainda que o fato de sermos generosos não nos usurpa nada, e que as maneiras mais eficazes de generosidade consistem em oferecermos nosso tempo, atenção, cuidado, suporte, experiência e conselhos.

Nesta mesma linha de raciocínio, Wilson [7] enfatiza que o processo evolutivo alcança mais sucesso em sociedades colaborativas com forte predominância da generosidade, ou seja, com o olhar voltado para o outro. Não há ônus, apenas bônus ao ajudar o próximo. Uma frase preocupante, mas digna de assinalar, é a do filósofo francês Compte-Sponville [8]: “A generosidade só brilha, na maioria das vezes, por sua ausência”. A generosidade e o altruísmo muitas vezes são ignorados; o chamado bem comum perde espaço para os interesses individuais.

\section{VOLUNTARIADO NAS ESCOLAS DE MEDICINA}

Em revisão da literatura referente às atividades voluntárias especificamente nas escolas de medicina, verificamos que a grande maioria das publicações descrevem atividades extracurriculares direcionadas para áreas técnicas, com mínima referência a atividades de voluntariado com caráter mais social e beneficente [9-16]. Entretanto, cientes da importância do voluntariado durante o curso de medicina, instituições de ensino como a Universidade do Porto, em Portugal, propõem a possibilidade de transformar essas atividades em uma disciplina dentro do currículo do curso [17].

Sussman e Cloninger [3], destacando que a generosidade é frequentemente ignorada em nossa sociedade, apontam para a necessidade de realizar 
atividades que potencializem a capacidade dos estudantes de se tornarem mais abertos para as diferenças e generosos nas relações humanas em suas futuras atividades profissionais. Apesar dos benefícios dessas atividades, cabe a reflexão sobre o quanto estamos dispostos a considerá-las como atividades oficialmente curriculares. De forma mais objetiva e cética, de que forma podemos avaliar os benefícios reais dessas ações para as escolas de medicina?

Sem que tenham resposta para todas as dúvidas e questionamentos, Smith e Davidson [18] destacam algumas vantagens de quem se dedica ao voluntariado. Segundo relatam esses autores, nessas pessoas o nível de felicidade é em média 7\% superior ao grupo controle de não voluntários. Em relação às condições de saúde física os voluntários apresentaram uma média de qualidade de vida $13 \%$ superior ao grupo controle [18].

Outro beneficio das atividades de voluntariado é a possível participação das famílias dos alunos nas ações realizadas, podendo aproximar estudantes e suas famílias dos projetos curriculares e extracurriculares. Um artigo evidenciou que jovens que tiveram o exemplo e apoio familiar tiveram um índice de $18 \%$ a mais em termos de engajamento com voluntariado e atos de generosidade, comparados aos controles sem suporte familiar [18].

Toguetta [19] lembra os Filósofos Luc Ferry e Compte-Sponville, que afirmam que o ser humano busca as virtudes por sua própria natureza. Hamilton [20] reforça a teoria de que os atos de generosidade conduzem a um grau maior de felicidade. Essa ligação funciona como um gatilho para que o cérebro produza endorfinas, o que leva a um estado de euforia parecido com o efeito produzido pela morfina, todavia de forma mais atenuada. $\mathrm{O}$ autor denomina esse estado de helper's high, relacionado ao aumento do nível de serotonina e dopamina no cérebro. No momento em que são realizados atos de bondade e generosidade há também liberação de ocitocina, substância que é considerada inclusive cardioprotetora, podendo resultar em diminuição dos níveis tensionais e melhor eliminação de radicais livres. Destaca, ainda, a possibilidade da melhora da função imunológica, lembrando pesquisa onde era referido um índice de $13 \%$ de melhores condições de saúde no grupo de voluntários em ações beneficentes [20].

É impossível analisar estes dados e não pensar no mundo moderno, onde milhões de pessoas buscam ajuda em medicamentos, livros ou programas de autoajuda na busca de alguns momentos de "felicidade". Em seu livro, Post [21] também destaca que pensar no outro e em suas necessidades diminui o nível de estresse e ansiedade, além de ativar áreas do cérebro responsáveis pela liberação de dopamina.

Projetos de Voluntariado da Pontifícia Universidade Católica do Rio Grande do Sul (PUCRS) vêm sendo realizados pelo Centro de Pastoral e Solidariedade da universidade. Os objetivos desses projetos incluem "viabilizar experiências de voluntariado como meio para despertar valores humanos, buscando a formação de agentes de transformação social; proporcionar a formação continuada aos voluntários na perspectiva da solidariedade, do exercício da cidadania e no desenvolvimento da consciência crítica sobre a realidade social local e do país; e promover a sensibilização para a participação cidadã permanente e a integração comunitária em busca do bem comum". As áreas de atuação dos voluntários são Saúde, Educação e Assistência Social, e os participantes podem ser universitários, técnicos administrativos, professores ou diplomados [22,23].

No Programa de Voluntariado da Escola de Medicina da PUCRS, criado em 1995, os alunos têm oportunidade de estreita relação com adultos e crianças de comunidades carentes. As pessoas atendidas não são tratadas como pacientes, pois na maioria das vezes as ações desenvolvidas não são atividades médicas, ou seja, não possuem caráter clínico. Sob orientação de professores, os alunos realizam, por exemplo, eventos em conjunto com lideranças comunitárias locais, para festejar datas como Páscoa, Dia das Mães e outras. Por adesão voluntária e em reuniões periódicas, os estudantes organizam campanhas de arrecadação de brinquedos ou brindes, ajudam a arrumar os espaços comunitários e preparam brincadeiras com as crianças e atividades com os adultos. Um evento tradicional é o realizado por ocasião do Dia da Criança, em parceria com o Museu de Ciências e Tecnologia da PUCRS (que mantém um programa permanente de gratuidade para crianças de comunidades de baixa renda). Durante essa atividade, cada estudante de medicina torna-se responsável por uma das crianças visitantes. Antes de iniciar a visita ao museu, cada voluntário abraça o seu "afilhado" e entrega um presente, ao mesmo tempo em que é tirada uma fotografia para recordação. A seguir, todo o grupo participa do café da manhã patrocinado pela Escola de Medicina e, após, as crianças são levadas a conhecer o museu, com especial atenção às atrações que tratam de biologia, saúde e funcionamento do corpo humano, nas quais os estudantes de medicina têm oportunidade de fornecer às crianças diversos ensinamentos importantes, mas de uma forma lúdica. 
Nessas atividades de voluntariado notam-se reações ligadas à empatia, quando a ajuda a outras pessoas é feita "face a face", de forma presencial. A participação em atividades de apoio a outros indivíduos ou a dedicação a um trabalho voluntário por algumas horas semanais parecem estar associadas a um evidente aumento do grau de felicidade, o qual é relatado pelos estudantes voluntários. Há indícios de melhora na saúde física, baixa dos níveis de ansiedade, diminuição do estresse e melhora na qualidade do sono. Observações similares podem ser encontradas na publicação de Wilson e Musick [24].

\section{CONCLUSÕES}

As atividades de voluntariado durante o curso de medicina podem proporcionar ao estudante o contato direto com comunidades e pessoas, especialmente aquelas em condições de vulnerabilidade social, promovendo um maior envolvimento humano, refletido em gestos e atitudes que trazem benefícios mútuos, a quem presta e a quem recebe.

Voluntariado voltado às ações de generosidade e beneficência pode ser resumido em uma frase: se eu faço pensando em você, isso é generoso. Ser generoso é ter um olhar mais amplo, que enxerga mais longe e supera eventuais diferenças e dificuldades. É conseguir ter empatia, colocar-se no lugar do outro, entendendo seu sofrimento e suas necessidades. É poder, sem abrir mão de crenças e princípios, ajudar a quem precisa. É conseguir romper as amarras egoístas e narcisistas que nos fazem focar apenas em nossas próprias questões. Além disso, é estar aberto para mudar o tempo todo, para reinventar-se. Poder ajudar e permitir que sejamos ajudados e, com isso, abrir espaços, criar redes de colaboração e solidariedade, são condições ou virtudes necessárias ao bom desempenho da medicina.

\section{NOTA}

Declaração de conflitos de interesse

Os autores declaram não haver conflitos de interesse relevantes ao conteúdo deste estudo.

\section{REFERÊNCIAS}

1. Houaiss A. Dicionário da Língua Portuguesa. Rio de Janeiro: Objetiva; 1999.

2. United Nations Organization. UN Volunteers. The power of volunteerism [Internet]. [Cited 2016 Oct 30]. Available from: https://www. unv.org/volunteerism

3. Sussman RW, Cloninger CR (eds.). Origins of altruism and cooperation. London: Springer; 2011.

4. Van Dyken JD, Wade MJ. Origins of altruism diversity I: the diverse ecological roles of altruistic strategies and their evolutionary responses to local competition. Evolution. 2012;66(8):2484-97. http://dx.doi.org/10.1111/j.1558-5646.2012.01630.x

5. Van Dyken JD, Wade MJ. Origins of altruism diversity II: Runaway coevolution of altruistic strategies via "reciprocal niche construction". Evolution. 2012;66(8):2498-513. http://dx.doi.org/10.1111/j.1558-5646.2012.01629.x

6. Burg B, Mann JD. O Conselheiro. Rio de Janeiro: Ed. Sextante; 2010.

7. Wilson EO, Korytowski I. A conquista social da terra. São Paulo: Companhia das Letras; 2013.

8. Comte-Sponville A. Pequeno tratado das grandes virtudes. São Paulo: Martins Fontes; 2009.

9. Margarido MR. Atividades extracurriculares, uma opinião. Medicina (Ribeirão Preto). 2013;46(1):56-8. http://dx.doi.org/10.11606/ issn.2176-7262.v46i1p56-58

10. Chehuen Neto JA, Sirimarco MT, Cândido TC, Ferreira IdA, Campos RCF, Martins SC. Currículo paralelo na graduação médica na perspectiva dos estudantes. Rev Méd Minas Gerais. 2013;23(4):467-78. http://rmmg.org/artigo/detalhes/409

11. Marques, VL. Voluntariado: motivos e repercussões na vida pessoal, social e acadêmica dos alunos de graduação em medicina, voluntários em programas na área da saúde [dissertation]. [São Paulo]: Universidade de São Paulo; 2006. http://www.dominiopublico.gov.br/pesquisa/ DetalheObraForm.do?select_action=\&co_obra $=41105$

12. Tavares CHF, Maia JA, Muniz MCH, Malta MV, Magalhães BRdC, Thomaz ACP. O currículo paralelo dos estudantes da terceira série do curso médico da Universidade Federal de Alagoas. Rev Bras Educ Méd. 2007;31(3):245-53. http://dx.doi.org/10.1590/s010055022007000300007

13. Vieira EM, Barbieri CLA, Vilela DB, Júnior EI, Tomé FS, Woida FM, Maia F, Martinez GL, Vicente LM, Gava NF, Lira PG, Brandão TO, Mendonça TN. O que eles fazem depois da aula? As atividades extracurriculares dos alunos de ciências médicas da FMRP-USP. Medicina (Ribeirão Preto). 2004;37(1/2):84-90. http://www.revistas.usp.br/rmrp/article/view/480

14. Peres CM, Andrade AS, Garcia SB. Atividades extracurriculares: multiplicidade e diferenciação necessárias ao currículo. Rev Bras Educ Méd. 2007;31(3):203-11. http://dx.doi.org/10.1590/s0100-55022007000300002 
15. Roulin N, Bangerter A. Extracurricular activities in young applicants' résumés: What are the motives behind their involvement? Int $\mathbf{J}$ Psychol. 2013;48(5):871-80. http://dx.doi.org/10.1080/00207594.2012.692793

16. Pinheiro da Costa BE, Hentschke MR, Cruz da Silva AC, Barros A, Salerno M, Poli-de-Figueiredo CE, et al. Reflexões sobre a importância do currículo informal do estudante de medicina. Sci Med. 2012;22(3):162-8. http://revistaseletronicas.pucrs.br/ojs/index.php/scientiamedica/ article/view/10052

17. Magalhães O. Voluntariado passa a ser cadeira na Faculdade de Medicina do Porto, Portugal [Internet]. Porto, Portugal; 6 de outubro, 2014 [Cited 2016 Oct 30]. Available from: https://noticias.up.pt/voluntariado-passa-a-ser-cadeira-na-faculdade-de-medicina/

18. Smith C, Davidson H. The paradox of generosity: Giving we receive, grasping we lose: Oxford University Press, USA; 2014. http://dx.doi org/10.1093/acprof:oso/9780199394906.001.0001

19. Tognetta LRP. Perspectiva ética e generosidade. Campinas: Mercado de Letras; 2009.

20. Hamilton DR. Why kindness is good for you. Carlsbad, CA: Hay House Inc; 2010.

21. Post S, Neimark J. Why good things happen to good people: How to live a longer, healthier, happier life by the simple act of giving. New York: Broadway Books; 2007.

22. Pontifícia Universidade Católica do Rio Grande do Sul. Voluntariado PUCRS [Internet]. [Cited 2016 Oct 30]. Available from: http://www. pucrs.br/voluntariado/

23. Pontifícia Universidade Católica do Rio Grande do Sul. Centro de Pastoral e Solidariedade PUCRS [Internet]. [Cited 2016 Oct 30 ]. Available from: http://www.pucrs.br/pastoral/

24. Wilson J, Musick M. The effects os volunteering on the volunteer. Law Contemp Probl. 1999;62(4):141-68. http://scholarship.law.duke. $\mathrm{edu} / \mathrm{lcp} / \mathrm{vol} 62 / \mathrm{iss} 4 / 7$ 